University of Texas Rio Grande Valley

ScholarWorks @ UTRGV

Psychological Science Faculty Publications and

Presentations

College of Liberal Arts

$8-2020$

\title{
The Relationship between Sexual Orientation Outness, Heterosexism, Emotion Dysregulation, and Alcohol Use among Lesbian, Gay, and Bisexual Emerging Adults
}

\author{
Lillianne Villarreal \\ The University of Texas Rio Grande Valley \\ Ruby Charak \\ The University of Texas Rio Grande Valley, ruby.charak@utrgv.edu \\ Rachel M. Schmitz \\ Oklahoma State University \\ Claire Hsieh \\ The University of Texas Rio Grande Valley \\ Julian D. Ford \\ University of Connecticut Health Center \\ Follow this and additional works at: https://scholarworks.utrgv.edu/psy_fac \\ Part of the Psychology Commons
}

\section{Recommended Citation \\ Villarreal, L., Charak, R., Schmitz, R. M., Hsieh, C., \& Ford, J. D. (2020). The Relationship between Sexual Orientation Outness, Heterosexism, Emotion Dysregulation, and Alcohol Use among Lesbian, Gay, and Bisexual Emerging Adults. Journal of Gay and Lesbian Mental Health. Advance online publication. doi.10.1080/19359705.2020.1809588}

This Article is brought to you for free and open access by the College of Liberal Arts at ScholarWorks @ UTRGV. It has been accepted for inclusion in Psychological Science Faculty Publications and Presentations by an authorized administrator of ScholarWorks @ UTRGV. For more information, please contact justin.white@utrgv.edu, william.flores01@utrgv.edu. 
The Relationship between Sexual Orientation Outness, Heterosexism, Emotion

Dysregulation, and Alcohol Use among Lesbian, Gay, and Bisexual Emerging Adults

\author{
Lillianne Villarreal, B.S. \\ School of Medicine, University of Texas Medical Branch, Galveston, TX \\ Ruby Charak, Ph.D. \\ Department of Psychological Science, The University of Texas Rio Grande Valley, Edinburg, \\ TX \\ Rachel M. Schmitz, Ph.D. \\ Department of Sociology, Oklahoma State University, Stillwater, OK \\ Claire Hsieh, B.S. \\ Department of Psychological Science, The University of Texas Rio Grande Valley, Edinburg, \\ TX. \\ Julian D. Ford, Ph.D. \\ Department of Psychiatry, University of Connecticut Health Center, CT
}

Corresponding author: Ruby Charak, PhD, Assistant Professor, Department of Psychological

Science, The University of Texas Rio Grande Valley, EIEAB 3.207, 1201 W University Dr.,

Edinburg, TX. Ph: +1-9566653733. Email: charakruby@gmail.com

Please cite as (APA citation): Villarreal, L., Charak, R., Schmitz, R. M., Hsieh, C., \& Ford, J. D. (2020). The Relationship between Sexual Orientation Outness, Heterosexism, Emotion Dysregulation, and Alcohol Use among Lesbian, Gay, and Bisexual Emerging Adults. Journal of Gay and Lesbian Mental Health. Advance online publication. doi.10.1080/19359705.2020.1809588 


\begin{abstract}
INTRODUCTION: The current cross-sectional study investigated a sequential mediation model wherein heterosexist experiences (HE) and emotion dysregulation (ER) were hypothesized to mediate the relation between outness and alcohol use in lesbian, gay, and bisexual (LGB) emerging adults.

METHOD: Participants were 264 LGB in the age range of $18-29$ years $(M / S D=25.46 / 2.74$; $16.7 \%$ lesbian, $23.1 \%$ gay, $60.2 \%$ bisexual).

RESULTS: Findings linked lower levels of outness with harmful alcohol use via HE and ER, with a significant serial indirect effect and indirect effects via both HE and ER.

DISCUSSION: Clinical interventions should be directed toward reducing HE and in developing emotion regulation skills among those at risk of harmful alcohol use.
\end{abstract}

Keywords. lesbian, gay, bisexual, emerging adults, outness, emotion dysregulation, heterosexism, alcohol use 


\section{The Relationship between Sexual Orientation Outness, Heterosexism, Emotion Dysregulation, and Alcohol Use among Lesbian, Gay, and Bisexual Emerging Adults}

Patterns of alcohol use in the United States among sexually diverse individuals show that compared to their heterosexual counterparts, lesbian, gay, or bisexual (LGB) individuals report higher rates of alcohol use and are at increased risk of developing alcohol use disorders (Allen \& Mowbray, 2016; Charak, Villarreal, Schmitz, Hirai, \& Ford, 2019; Gonzales, Przedworski, \& Henning-Smith, 2016). A meta-analysis of prevalence rates of mental disorder and substance use in LGB individuals found that the risk of developing alcohol dependence was 1.5 times higher in sexually diverse individuals than in heterosexuals (King et al., 2008). In addition to increased alcohol use, LGB individuals also reported increased rates of harmful patterns of alcohol consumption, such as heavy episodic drinking and alcohol use at a young age (Bryan et al., 2017; Burgard, Cochran, \& Mays, 2005; Gruskin, Hart, Gordon, \& Ackerson, 2001; King et al., 2008; Wilsnack et al., 2008). Furthermore, the developmental stage of emerging adulthood (18-29 years; Arnett, 2000, 2004, 2011) is a particularly vulnerable period, with prior studies indicating the highest rates of alcohol use and binge drinking (i.e., consuming four/five or more drinks on one occasion) among this age group (Esser et al., 2014; Kanny, Naimi, Liu, \& Lu, 2019). A recent longitudinal cohort study on emerging adults indicated a higher likelihood of alcohol use disorders among sexually diverse individuals compared to their heterosexual peers (Coulter et al., 2018). Thus, it is important to examine the multiple risk factors that may contribute to harmful alcohol use among LGB emerging adults, which will be the focus of the present study.

Specific to LGB individuals, a unique and proximal personal characteristic associated with behavioral health disorders, including harmful alcohol consumption, is the degree of outness regarding one's sexual orientation. Broadly, sexual orientation outness refers to the 
levels of openness one exhibits surrounding both their sexual identity concealment and disclosure practices within their social networks (Meidlinger \& Hope, 2014). Findings, however, are mixed with some studies indicating that a higher degree of outness results in diminished psychological distress (Juster, Smith, Ouellet, Sindi, \& Lupien, 2013; Morris, Waldo, \& Rothblum, 2001), while other studies suggest that increased outness is instead tied to poorer mental health outcomes (Pachankis, Cochran, \& Mays, 2015; Rosario, Schrimshaw, \& Hunter, 2009).

Prior studies also indicate that elevated alcohol use is related to distal identity-related stressors, such as stigmatizing experiences of discrimination surrounding an LGB individual's marginalized sexual identity, known as heterosexism (Lee, Gamarel, Bryant, Zaller, \& Operario, 2016; Livingston, Christianson, \& Cochran, 2016; McCabe, Bostwick, Hughes, West, \& Boyd, 2010). Exposure to heterosexism can be a source of significant distress for LGB individuals (Swim, Johnston, \& Pearson, 2009; Szymanski \& Mikorski, 2016), and may be linked to another proximal individual characteristic that is associated with unhealthy alcohol use - emotion dysregulation (Tice, Bratslavsky, \& Baumeister, 2001; Veilleux, Skinner, Reese, \& Shaver, 2014).

Taken together, the mixed findings across studies focusing on outness and alcohol use suggest underlying mechanisms that may help explain these factors' interrelationship. However, there are only a handful of studies that test potential mediators in the relationship between LGB outness and mental health challenges (Hughes, 2011; Rogers et al., 2017) and notably, these studies examine the role of potential linkages separately rather than in combination. Identifying factors that elevate the risk of harmful alcohol use among LGB individuals is crucial as this will aid in developing appropriate interventions and possibly mitigate the negative consequences 
associated with harmful alcohol consumption. Thus, the current cross-sectional study aimed to bridge the gap in the literature by testing a sequential integrated model to examine the serial mediating role of heterosexism and emotion dysregulation in the association between outness regarding one's minority sexual orientation and harmful alcohol use in LGB emerging adults.

\section{LGB Minority Stressors, Outness, and Alcohol Use}

As a marginalized social group, sexually diverse individuals are subject to prejudice, discrimination, and stigma — at significantly higher rates than their heterosexual counterparts (Mays \& Cochran, 2001) — related to their identification as lesbian, gay, or bisexual (Meyer, Schwartz, \& Frost, 2008). These societal stressors shape elevated levels of stress among LGB people and can exert negative influence on an LGB individual's mental health (Schmitz, Robinson, Tabler, Welch, \& Rafaqut, 2019). Specifically, minority stress theory (MST) examines the unique stressors endured by LGB individuals (Meyer, 2003). Synthesizing elements from various social and psychological theories, mainly the social stress theory (Crocker, Major, \& Steele, 1998), MST elucidates the marginalizing conditions and mechanisms surrounding the elevated rates of negative psychological consequences among LGB people (King et al., 2008). MST proposes that LGB individuals experience four processes, namely, external stressful events and conditions, expectations of rejection surrounding and related vigilance toward these events, internalized homophobia from negative societal views and concealment of one's sexual orientation, all of which serve as sources of distress. With reference to one's self, these stressors are arranged on a distal-proximal continuum, where proximal stressors are more subjective and largely rely on an LGB individual's self-identity, and distal stress processes are objective experiences independent of individual perceptions (Meyer, 2003). 
The minority stressors in MST, especially the proximal stressors of expectations of rejection, concealment, and internalized homophobia, are primarily stressors associated with outness, or openness about one's sexual identity (Meidlinger \& Hope, 2014). The current study focuses on outness, comprised of two interrelated albeit distinct, independent concepts: disclosure, which is the active verbal or physical indication of one's sexual orientation, and concealment, which involves actively avoiding such disclosure (Meidlinger \& Hope, 2014). LGB individuals actively engage in continuous management of their sexual identities and must strike a balance between how much, and to whom, they choose to conceal and/or disclose information surrounding their stigmatized sexual identity (Craig \& McInroy, 2014). These processes are an integral part of an LGB individual's healthy sexual identity development and are intricately linked to their well-being (Riggle, Rostosky, Black, \& Rosenkrantz, 2017; Schmitz \& Tyler, 2018a).

As discussed, research findings on the association between degree of outness and effects on mental health have produced contrasting results. These contradictory findings suggest that being out regarding one's sexual orientation is largely contextual and varies greatly depending on the presence of social support or experiences of rejection and victimization. For example, gay and bisexual men who were more open about their sexual orientation at their workplace reported higher levels of distress and negative affect (Huebner \& Davis, 2005). In the 2011 National School Climate Survey on the experiences of LGBT secondary school students, a higher degree of outness was positively related with a higher self-esteem and lower rates of depression. However, the same study also observed that increased disclosure from students, an important dimension within the concept of outness, was associated with elevated risk for adverse mental health outcomes (Kosciw, Greytak, Bartkiewicz, Boesen, \& Palmer, 2012). 
Specific to alcohol use, a higher degree of outness was a significant risk factor for consuming alcohol, especially among bisexual individuals (Feinstein, Dyar, \& London, 2017), and studies have linked negative reactions to a person's sexual orientation upon disclosure, an important facet of outness, to increased risk for alcohol use (Goldbach, Tanner-smith, Bagwell, \& Dunlap, 2014; Rosario et al., 2009). However, a significant association between degree of outness and alcohol consumption has not always been observed (e.g., Feinstein et al., 2019), indicating underlying processes that have yet to be identified.

\section{Heterosexism and its Association with Outness and Alcohol Use}

Distal, objective instances of sexual minority-specific discrimination in social and cultural contexts, referred to as experiences of heterosexism, suffuse various facets of an LGB individual's sexual identity, such as family relations and HIV/AIDS status (Balsam, Beadnell, \& Molina, 2013). Broadly, heterosexism includes social structures and ideologies that privilege heterosexual identities and behaviors by casting heterosexuality as superior and normative while subordinating diverse sexualities (Simoni \& Walters, 2001). The process of coming out has been identified as a coping mechanism in buffering the stress perceived from experiences of heterosexism, as LGB individuals anticipate and learn to combat their unique minority stressors (Meyer, 2003; Morris et al., 2001), thus preliminarily suggesting an association between outness and heterosexist experiences. In the 1994 National Lesbian Health Care Survey, being out was associated with diminished stress related to rejection and discrimination upon disclosure (Bradford, Ryan, \& Rothblum, 1994). Similarly among young adults, coming out in school contexts can promote more beneficial psychosocial well-being (Russell, Toomey, Ryan, \& Diaz, 2014). Outness may not always serve as a protective buffer against the effects of heterosexism, however, as sexually diverse individuals who were more out (i.e., reporting higher levels of 
disclosure and lower levels of concealment) also exhibited elevated stress associated with the distal minority stressors of victimization and discrimination (Balsam et al., 2013). Sexual orientation outness elevates visibility and can generally increase the likelihood of enduring sexual orientation-specific experiences of discrimination (Kosciw, Palmer, \& Kull, 2014), and these experiences may be a link between outness and adverse mental health outcomes.

Studies indicate that experiences of sexual minority-specific discrimination are associated with general psychological and emotional distress, anxiety, depression, and hazardous drinking, thus supporting the link between LGB status-related discrimination and negative mental health outcomes (Correro \& Nielson, 2019; Dyar, Newcomb, \& Mustanski, 2019; Hatzenbuehler, Corbin, \& Fromme, 2011; Ngamake, Walch, \& Raveepatarakul, 2016; Williams, Connolly, Pepler, \& Craig, 2005). For example, in a study conducted by Lewis and colleagues (2016) on lesbian women, the distal stressor of perceived sexual minority discrimination was significantly associated with negative health outcomes, including hazardous drinking, as mediated by maladaptive coping. Similarly, in their study on lesbian women, Dorn-Medeiros \& Doyle (2019) also reported that their participants used alcohol, described as having a "numbing" effect, to cope with experiences of heterosexism. This association between LGB status-based discrimination and alcohol use has also been observed in the youth, as one study among a sample of Australian youth aged 14-24 found that 58\% of respondents perceived their experiences of homophobia and discrimination as having an impact on their level of alcohol use (Kelly, Davis, \& Schlesinger, 2015).

\section{Challenges with Emotion Regulation and Alcohol Use}

While there is a link between LGB-minority stressors and increased alcohol use, not everyone with experiences of distal or proximal minority stressors demonstrates harmful alcohol 
consumption. LGB individuals commonly endure risk and/or develop resilience depending on a range of coping skills in response to experiences of heterosexism and the emotional distress associated with those experiences (Meyer, 2003). Adaptive coping mechanisms include individualistic coping strategies such as developing a positive personal identity (Schmitz \& Tyler, 2019) and problem-solving responses to stressors (Kaysen et al., 2014); group-level strategies such as greater social support are also particularly important for LGB people (Meyer, 2003; Riggle et al., 2017; Williams et al., 2005). When certain personal or group-level resources are not available to an individual, they may instead experience difficulties with emotion regulation, which includes the strategies an individual adopts to modulate their emotional responses to a stressful situation (Gross, 2001). Enduring heterosexist and anti-LGB discriminatory experiences can take its psychological toll over time on LGB people and increase the risk of developing psychopathology by eroding the capacity for emotion regulation (McLaughlin, Hatzenbuehler, \& Hilt, 2009), as posited by the psychological mediation framework (PMF; Hatzenbuehler, 2009).

Difficulties with emotion regulation, or emotion dysregulation, is the inability to manage the intensity and duration of negative emotions (e.g., fear, anger) in response to provocative stimuli. Studies indicate a robust association between emotion dysregulation and substance use (Aldao, Nolen-Hoeksema, \& Schweizer, 2010; Feinstein \& Newcomb, 2016). The selfmedication hypothesis suggests that individuals use substances, including alcohol, to manage stress (Khantzian, 1997). Within the general population, it is well-documented that difficulties with emotion regulation increase the risk of developing alcohol use disorders (Shadur \& Lejuez, 2015; Tice et al., 2001; Veilleux et al., 2014). This stems from the motivation to regulate and numb the negative emotions via excessive alcohol consumption (Cooper, Frone, Russell, \& 
Mudar, 1995), specifically in the presence of maladaptive emotion regulation strategies such as suppression and avoidance (Aldao et al., 2010; Nolen-Hoeksema \& Harrell, 2002).

Hatzenbuehler and colleagues (2009) have furthered Meyer's MST by positing via the PMF that sexual minority-specific stigma and increases in psychopathology among sexual minority people could be linked via emotion dysregulation (Aldao et al., 2010; McLaughlin, Hatzenbuehler, Mennin, \& Nolen-Hoeksema, 2011).

In line with this framework, studies have begun to identify emotion regulation as a coping strategy among LGB individuals that plays an important role in the association between distal minority stressors associated with LGB status (e.g., victimization and family rejection), the stress perceived from such experiences, and increased substance use (Hatzenbuehler, Nolenhoeksema, \& Dovidio, 2009; Rogers et al., 2017). Notably, emotion regulation skills predicted lower levels of alcohol use equally among LGB individuals, suggesting that emotion regulatory skills may be an effective target for clinical intervention (Rogers et al., 2017). However, there is little to no research exploring the relationship between emotion regulation and levels of outness independently of discriminatory experiences, and no study to date considers the roles of both difficulties in emotion regulation and experiences of heterosexism in potentially mediating degrees of outness and alcohol use among LGB individuals.

\section{The Current Study}

Based on MST (Meyer, 2003) and the self-medication hypothesis (Khantzian, 1997), prior studies, albeit independently, have demonstrated that minority stressors (e.g., outness, heterosexism) and general psychological stressors (e.g., difficulties in emotion regulation) lead to increased alcohol use among sexually diverse individuals. However, no study examines the serial mediating effect of heterosexism and emotion dysregulation operating between degree of outness 
and alcohol use. The current cross-sectional study aimed to bridge this gap in the literature by testing a sequential mediation model consistent with MST (Meyer, 2003) and the self-medication model (Khantzian, 1997) to explain the association between outness and alcohol use.

Specifically, the present study examined the indirect effect of distress from heterosexism and emotion dysregulation as mediators between outness regarding one's sexual orientation status and alcohol use among LGB emerging adults. In doing so, three indirect effects were examined focusing on (i) the indirect effect of perceived stress due to heterosexism, (ii) the indirect effect of emotion dysregulation, and (iii) the sequential indirect effect of perceived stress due to heterosexism and emotion dysregulation on the association between degree of outness and alcohol use. The present study first hypothesized that a lower degree of outness would be associated with higher alcohol use via increased perceived stress from heterosexism (Balsam et al., 2013; Bradford et al., 1994) and greater emotion dysregulation (Rogers et al., 2017), sequentially. Second, it was hypothesized that the strength of the serial indirect effect of perceived stress from heterosexism and emotion dysregulation would be significantly stronger than the remaining two indirect effects when operating between degree of outness and alcohol use. Therefore, lower degree of outness would lead to higher heterosexism-related perceived stress that in turn would lead to greater emotion dysregulation further leading to increase in alcohol use.

\section{Method}

\section{Participants}

Participants were 264 emerging adults ranging in age from 18 to 29 years $(M=25.46, S D$ $=2.74)$, consisting of $161(61.0 \%)$ women, $102(38.6 \%)$ men, and $1(0.4 \%)$ intersex individual. The inclusion criteria in the present study were (i) age range of 18-29 years, (ii) a United States 
citizen or legal resident, (iii) have at least one brother or sister, and (iv) have a current or former dating partner or spouse, and the exclusion criterion was self-identifying as other than LGB. Regarding sexual orientation self-identification, 61 (23.1\%) identified as gay, 44 (16.7\%) identified as lesbian, and $159(60.2 \%)$ identified as bisexual. The self-reported ethnic and racial backgrounds of the 264 participants were $57.2 \%$ White non-Hispanic, 25.4\% White Hispanic, 6.8\% Black or African American, 5.3\% Asian, 3.8\% Bi- or Multi-racial, and 1.5\% American Indian or Alaska Native. In terms of relationship status, $229(86.7 \%)$ were in an intimate relationship at the time of the study, with 54 (20.5\%) reporting being married, $45(17.0 \%)$ reporting cohabitation, and $130(49.2 \%)$ reporting being in a non-marital non-cohabiting intimate relationship, and $35(13.3 \%)$ were not in an intimate relationship at the time of the study but reported a past relationship.

\section{Procedure}

Participants completed an anonymous and incentivized online study on stressful life events and emotional experiences among LGB young adults. Participants were recruited online via Amazon's Mechanical Turk (Amazon MTurk), a sourcing platform considered a viable research participatory tool for gathering high-quality data from a diverse population (Buhrmester, Kwang, \& Gosling, 2011). Through MTurk, a pool of participants complete Human Intelligence Tasks (HITs) for compensation, such as research surveys. The Institutional Review Board (IRB) of the participating institution approved the study procedures. The current study was listed as a research survey link on Amazon MTurk and tagged with the keywords "survey," "psychology," "adverse life events," and "emotional problems." The study was advertised as one examining "the role of lifetime stressful events on emotional experiences among young adults." 
Data collection took place during July-August 2017. The HIT was available only to individuals who had an IP address located within the U.S. Interested participants could accept the HIT to access the link to the survey administered externally through Qualtrics and were then shown the IRB-approved consent form containing the study variables and the eligibility criteria. There was no mention that participants must self-identify as LGB in the consent form. If they consented, participants were taken directly to the survey and asked demographic questions, including sexual orientation (heterosexual, gay, lesbian, bisexual, other, e.g., asexual, pansexual, queer). If participants chose "heterosexual" or "other," the survey was not administered. They were taken to the end of the survey, thanked for their time and cooperation, and informed that they were not eligible for the study and that they would not be paid. Individuals who met all eligibility criteria and completed the survey were shown an IRB-approved debriefing form and a random 4-digit code was generated for each respondent for input on the MTurk HIT page to ensure that the survey was indeed fully and successfully completed. Following input of the code and subsequent completion of the HIT, participants were compensated $\$ 3.00$ by Amazon MTurk for their participation. To prevent reentry of a participant, every person who successfully completed the HIT received a "Qualification" to their unique Worker ID which indicated that they had previously completed the survey and prevented them from accessing another HIT with the survey. A total of 322 HITs were made available for completion. Of those, 22 were deemed invalid due to providing an invalid completion code following completion of the survey, and 12 respondents did not request compensation via MTurk and were thus excluded from the sample.

\section{Measures}

Degree of outness. The Nebraska Outness Scale (NOS; Meidlinger \& Hope, 2014) is a 10-item scale assessing an individual's degree of outness, or openness about one's sexual 
orientation, with an individual's parents, siblings, extended family, friends, coworkers or supervisors, and strangers across two related but independent subscales with 5 items each: concealment (NOS-C) and disclosure (NOS-D). The NOS total score and its subscales have been shown to have acceptable internal reliability, as well as discriminant, convergent, and predictive validity (Meidlinger \& Hope, 2014). Responses were rated on an 11-point Likert-type scale from $0(0 \%)$ to $10(100 \%)$, indicating the percentage of people of a specific group that are aware of an individual's sexual orientation for NOS-D and the percentage of time that participants avoided discussing their sexuality with each group for NOS-C, and responses were then summed. Higher scores on the NOS-D denote greater disclosure with others about their sexuality, whereas higher scores on the NOS-C denote greater concealment about their sexual orientation. A total score combining both subscales was created to measure overall degree of outness, where the NOS-C was reverse coded and the scores from each of the ten total items under the two subscales were summed and converted to a total outness score, ranging from 0 (indicating no disclosure and complete concealment) to 100 (signifying complete disclosure and no concealment). In the present study, Cronbach's alpha was .83 for the full-scale NOS score.

Heterosexist experiences. Unique minority stressors affecting LGB individuals were measured via the Daily Heterosexist Experiences Questionnaire (DHEQ; Balsam et al., 2013), a 50-item scale created for identifying and understanding LGB health disparities (e.g., "Having very few people you can talk to about being LGBT," "Being harassed in public because of your gender expression"). The DHEQ assesses minority stressors and perceived stress from such stressors across nine domains: gender expression, vigilance, parenting, discrimination and harassment, vicarious trauma, family of origin, HIV/AIDS, victimization, and isolation. Since inquiry about participants' children was not examined in the present study, the DHEQ parenting 
subscale was not included. The DHEQ has been shown to have acceptable internal reliability, as well as construct and concurrent validity (Balsam et al., 2013). Responses ranged from 0 , indicating that the experience had not occurred or was not applicable, to 5 (it happened, and it bothered me extremely), and the respective items under each subscale were added to determine the total scale score. Additionally, for descriptive statistics, a dichotomous variable $(0=a b s e n t$; $1=$ present ) was created to indicate experiences of heterosexism for each subscale; if a participant endorsed any item corresponding to each subscale (indicated by an answer of 1 or higher), heterosexist experiences of that type were considered present. In the present study, Cronbach's alpha $(\alpha)$ for the total score was $\alpha=.97$.

Emotion dysregulation. The 16-item version of the Difficulties in Emotion Regulation Scale (DERS-16; Bjureberg et al., 2016) is a self-report measure of an individual's typical levels of emotion dysregulation (ER; e.g., "I have difficulty making sense out of my feelings") across five areas: nonacceptance of emotional responses, difficulties engaging in goal-directed behaviors when distressed, difficulties controlling impulsive behaviors when distressed, lack of emotional awareness, limited access to effective strategies for emotion regulation, and lack of emotional clarity. The DERS-16 has retained excellent internal consistency when compared with the original 36-item scale (Fowler et al., 2014; Gratz \& Roemer, 2004) and has been shown to be psychometrically sound in clinical and community samples (Charak et al., 2019a; Miguel, Giromini, Colombarolli, Zuanazzi, \& Zennaro, 2017). Emotion regulation challenges were rated on a 5-point Likert-type scale, ranging from 1 (almost never) to 5 (almost always) and responses were summed. In the present study, Cronbach's alpha for each of the five subscales in the DERS16 was acceptable $(\alpha \mathrm{s}=.88$ to .92$)$. 
Alcohol use problems. Alcohol use problems were measured via the Alcohol Use Disorders Identification Test (AUDIT; Saunders et al., 1993), which assesses drinking behavior, adverse reactions, and problems experienced in the past twelve months. Alcohol use problems were rated on a 5 -point Likert-type scale $(0=$ never; $4=$ daily or almost daily $)$ and summed. A cut-off score of 8 on alcohol use on the AUDIT scale indicates harmful alcohol consumption (Saunders et al., 1993). In the present study, Cronbach's alpha was .79.

\section{Statistical Analyses}

Using IBM SPSS version 25, bivariate correlations were first conducted to test the association between outness, heterosexism, difficulties in emotion regulation, and alcohol use problems.

Second, using the PROCESS macro (Hayes, 2013) in (SPSS version 25), a serial mediation analysis (model 6) was carried out to evaluate the indirect effects of outness on the dependent variable of alcohol use, through the mediating variables of experience of heterosexism and difficulties in emotion regulation. All variables were treated as manifest/ observed variables. The magnitude of the indirect effects was examined using the product-of-coefficient approach to calculate standard errors of the indirect effects. The coefficient of the indirect effect is divided by its standard error and compared to a critical value with a z-test. As recommended by Preacher and Hayes (2008), bias-corrected bootstrapping procedures for confidence intervals with a total of 5,000 bootstrapped samples were used to corroborate findings from the product-of-coefficient tests. Use of bootstrapping method is recommended over the traditional causal steps approach, as the former has higher power while maintaining reasonable control over the Type I error rate (Mackinnon, Lockwood, \& Williams, 2004). In the present study, a 95\% confidence interval not containing a zero was considered statistically significant. Third, all significant specific indirect 
effects were compared with each other to examine differences between parameters under consideration (each pair of statistically significant indirect effects) using bootstrapping ( $n=$ 5,000 iterations).

\section{Results}

Bivariate correlations demonstrated that all study variables were significantly correlated $(p<.05)$ except for outness and alcohol use (Table 1). The magnitude of the correlation coefficients was low to moderate in magnitude. Figure 1 depicts the serial mediation model with all pathways reaching a significant level of $p<.05$.

In relation to participants' levels of outness, $97 \%$ of the participants reported disclosure of any aspect of their LGB identity to at least one of the five specified groups (i.e., immediate family, extended family, friends and acquaintances, coworkers and supervisors, or strangers) and 97.3\% reported any degree of concealment from at least one group, indicative of the interrelated but independent nature of both outness dimensions. More than half of participants reported some amount of disclosure to all five groups and $93.6 \%$ disclosed at least to friends and acquaintances; this group also prompted the highest degree of disclosure and the lowest degree of concealment from participants. Focusing on concealment, $61.7 \%$ of participants concealed to some degree from all five groups, with participants most often choosing to conceal from coworkers and supervisors. Average percentages of participants' disclosure and concealment across all five groups were $47.3 \%$ and $43.4 \%$, respectively, and the average for participants' overall degree of outness was 52 percent.

About $92.4 \%$ of participants reported experiencing any form of heterosexism, and nearly a quarter of these participants reported at least one heterosexist experience from each of the eight DHEQ subscales. The domains of heterosexism reported by more than half of the sample of 
participants were discrimination based on gender expression, experiences of vicarious trauma (i.e., exposure to reports of traumatic life events experienced by others), discrimination by family of origin, and distress related to HIV/AIDS. Under the domain of vicarious trauma, $78.8 \%$ of participants, the highest endorsement of any heterosexist experience, reported hearing about hate crimes that happened to LGBT people the participants did not know, and this experience was also the endorsed item that most often extremely bothered participants. Findings indicated that $8.3 \%$ of people $(n=22)$ scored higher than the cut-off score of 8 on the AUDIT scale $(M=2.78$, $S D=3.95)$, indicative of harmful alcohol consumption.

In the serial mediation model, the total effect was statistically significant (Table 2). The serial mediation model that included heterosexist experiences (HE) and difficulties in emotion regulation (DERS) as the mediators comprising three indirect effects (Outness $\rightarrow \mathrm{HE} \rightarrow$ Alcohol use; Outness $\rightarrow$ DERS $\rightarrow$ Alcohol use; Outness $\rightarrow$ HE $\rightarrow$ DERS $\rightarrow$ Alcohol use) were estimated as product of regression coefficients in the association between degree of outness and alcohol use. All three indirect effects were significant (Table 2) and indicated an inverse relation between degree of outness and alcohol use. Furthermore, the comparison of indirect effects indicated that the serial mediation effect of heterosexist experiences and difficulties in emotion regulation had a significantly stronger indirect (mediation) effect (Outness $\rightarrow \mathrm{HE} \rightarrow \mathrm{DERS} \rightarrow$ Alcohol use) than the individual indirect effect of heterosexism experiences and difficulties in emotion regulation (Outness $\rightarrow$ HE $\rightarrow$ Alcohol use $(z=.005, p<.05)$; Outness $\rightarrow$ DERS $\rightarrow$ Alcohol use $(z=.007, p<$ .05). No difference was found between the individual indirect effect of HE and DERS on alcohol use $(z=.001, p=.96)$.

\section{Discussion}


The primary purpose of the present study was to investigate, via a sequentially integrated model of minority stress theory (Meyer, 2003) and the self-medication hypothesis (Khantzian, 1997), the association between a proximal minority stressor, namely, one's degree of outness, and alcohol use via distal minority stress process of heterosexist experiences, and difficulties in emotion regulation in a sample of LGB emerging adults. Harmful alcohol consumption was reported by nearly $9 \%$ of the LGB emerging adults. On average, participants reported a degree of outness of $52 \%$, and a lower degree of outness was associated with greater perceived stress from experiences of heterosexism, as well as with higher levels of emotion dysregulation, and greater likelihood of harmful alcohol use (hypothesis 1). Notably, there was no significant direct association between outness and alcohol use, which is in line with previous studies assessing this association (Feinstein et al., 2019). Furthermore, in comparing the three indirect effects, there was support for the second hypothesis in that the strength of the sequential indirect effect of perceived stress due to heterosexism and emotion dysregulation was greater than their individual indirect effects on the association between outness and alcohol use problems. Findings from the present study are important in elucidating the nuances of mental health challenges among the marginalized population of LGB young adults, particularly in how minority stress can shape problematic alcohol consumption.

Study findings indicated that a lower degree of outness was related to increased alcohol use via three indirect pathways. In the first pathway, perceived stress as a result of heterosexism mediated the association between degree of outness and alcohol use, in that greater perceived stress from heterosexist experiences led to an increase in alcohol use with lower degree of outness. The proximal minority stress associated with low levels of sexual orientation outness and the distal minority stressor of heterosexism have both, as individual constructs, been 
implicated in increasing the risk of developing psychopathology, including increase in alcohol consumption among sexually diverse individuals (Hatzenbuehler et al., 2011; Rosario et al., 2009; Szymanski, 2009). One prior study among lesbian women indicated that disclosure of one's sexual orientation acts as a potential buffer against the negative psychological outcomes associated with instances of sexual minority-specific discrimination or heterosexism (Bradford et al., 1994). The present findings are in line with this study in that degree of outness was inversely related to alcohol use problems via heterosexist experiences. This particular finding may be due to the distress associated with a heightened fear of exposure (and potentially less disclosure and more concealment) in a context of heightened heterosexist discrimination and stigma. The increase in concealment and reduction in disclosure in a heterosexist context could lead to an increase in alcohol use among LGB young adults as an attempt to self-medicate the distress elicited by discrimination and stigma (Khantzian, 1997), as well as due to perceiving that seeking formal behavioral health services may not be a viable option (Coker, Austin, \& Schuster, 2010; Macapagal, Bhatia, \& Greene, 2016). Whether a low degree of outness in the context of heterosexism leads to attempts to self-medicate and diminished access to and acceptability of behavioral health services warrants investigation in future studies.

The second indirect pathway indicated that with greater levels of emotion dysregulation, there was an increase in the use of alcohol tied to a lower degree of outness. The role that emotion dysregulation plays in influencing negative mental health outcomes in sexually diverse individuals has been studied extensively (Charak et al., 2019b; Pachankis et al., 2015; Rogers et al., 2017), although the unique contribution of outness to this association, especially in predicting alcohol consumption, was lacking in previous studies. Based on the self-medication hypothesis, present findings suggest that LGB individuals may use alcohol in an attempt to self-medicate 
when experiencing emotional dysregulation, and that a lower degree of outness was associated with difficulties in emotion regulation. It is possible that LGB individuals who are more willing to disclose and less prone to concealment may experience diminished emotional distress and a stronger sense of emotion regulation in the face of distress. However, it also is possible that LGB individuals who have stronger emotion regulation skills (or greater social support for their sexual identity and a greater sense of emotion regulation as a result), may be more likely to choose to disclose and not conceal, and this may lead to a reduced risk or degree of problems with alcohol use. These considerations warrant further testing of the different directional possibilities underlying this indirect pathway from outness to alcohol use problems via emotion dysregulation in future research.

When considering the effect of the two mediating variables - perceived distress due to heterosexist experiences and difficulties in emotion regulation-in a sequence, the indirect effect was significant and of greatest strength. Specifically, lower degree of outness operated via greater perceived stress from heterosexism and greater difficulties in emotion regulation, which led to increased alcohol consumption, demonstrating the contextual nature of outness processes and the mental health disparities associated with minority stress (Schmitz \& Tyler, 2018b). Although independently, prior studies have demonstrated that distress due to heterosexist experiences and difficulties in emotion regulation (Tice et al., 2001; Veilleux et al., 2014) are related to harmful alcohol use. The present study expands these understandings by highlighting that the sequential combination of heterosexism and emotion dysregulation are associated with lower outness and higher levels of alcohol use in a cross-sectional design. Based on the comparison of indirect effects, the present findings suggest more complex processes than previously identified, as both minority stressors (e.g., outness, heterosexism) and general 
psychological processes (e.g., difficulties in emotion regulation) lead to an increase in alcohol use among those with lower levels of outness, albeit in a sequential manner. This combination provides a potential sociological and psychological explanation for the context in which lower levels of outness may be linked to increases in alcohol use.

These findings are important from an intervention standpoint in that emotion dysregulation can be targeted through individual therapy and clinical interventions should be directed toward developing emotion regulation skills among those at increased risk of using alcohol via exposure to heterosexism. Additionally, as the present study consisted of emerging adults between 18 and 29 years old, findings indicate that the importance of this formative developmental period should be affirmed and addressed by clinicians in clinical interventions (Hatzenbuehler \& Pachankis, 2016). Minority stressors such as heterosexism, however, require societal level interventions in the form of awareness programs, and the creation of institutional policies will prove efficacious at protecting LGB individuals from harassment and victimization based on their sexual identity (Link \& Hatzenbuehler, 2016). For example, an LGB-supportive workplace climate that promotes LGB employees (e.g., pay, job promotion, performance) and not merely the absence of heterosexism enhances job satisfaction (Velez \& Moradi, 2016) and in turn may support LGB people's overall wellbeing.

\section{Limitations}

The present study findings should be considered within the context of the following limitations. First, the present study used a convenience sample of self-identifying as LGB (of which the majority were White non-Hispanic bisexual women) recruited through an online forum and may not be representative of all LGB emerging adults. A characteristic of this sample, central to the associations being investigated, was self-identification as a lesbian, gay, or bisexual 
individual; however, there was a lack of inclusion of other minority sexual orientations (e.g., pansexual, asexual) due to a lack of participants identifying as them, limiting the generalizability of the results to the vast membership of sexually diverse communities. Second, the present study was conducted through an online data collection platform (i.e., using Amazon MTurk), which may limit the generalizability of the study's findings to other samples (Walters, Christakis, \& Wright, 2018). However, online studies have been found comparable to college samples (Buhrmester et al., 2011) and studies show that MTurk participants consistently provide accurate, reliable data, and that the anonymity afforded to participants makes them more comfortable and willing to disclose sensitive information (Shapiro, Chandler, \& Mueller, 2013; Thomas \& Clifford, 2017). Third, the cross-sectional design of the present study limits conclusions regarding the temporal associations among variables and the assumption that perceived stress from heterosexism leads to emotion dysregulation. Future studies should examine the present model in a longitudinal design to establish the temporal relation between study variables. Fourth, the outness variable combined both disclosure and concealment, which may reflect different processes and may therefore have different associations with the proposed mediators and alcohol use outcome. Examination of disclosure and concealment separately is warranted in future studies to determine whether these two aspects of outness have similar or different relations to minority stress, emotion dysregulation, and behavioral health outcomes. Finally, harmful alcohol use was assessed with a brief screening measure that, while well validated, may not capture the full range and impairment due to unhealthy alcohol use - and other behavioral health outcomes (e.g., drug use, depression) warrant study. Additionally, the cut-off scores used in the present study was based on a general population sample and future 
studies should investigate the cut-off score for harmful alcohol use in a representative sample of LGB adults.

\section{Conclusion}

With prior studies suggesting that LGB individuals are at a higher risk of alcohol use compared with heterosexual individuals (e.g., Coulter et al., 2018), it is critical to identify unique stressors among LGB populations that elevate their risk for alcohol consumption. Although a few such stressors were identified in this study, namely, heterosexist experiences and emotion dysregulation associated with degree of outness, future work should investigate additional factors potentially operating in the association between outness and alcohol, such as the roles of social support, internalized heterosexism, and positive LGB identity (Chow \& Cheng, 2010; Whitman \& Nadal, 2015). Continued research on the unique proximal and distal stressors impacting LGB people can mitigate the resulting negative psychological consequences via awareness programs and clinical interventions that address the potential mechanisms identified through mediation analyses (e.g., Dialectical Behavior Therapy for emotion dysregulation). Furthermore, clinical services should be specifically tailored to address minority stressors within different groups of LGB individuals, such as LGB people of color or the specific subgroups of LGB communities, and properly integrate various minority stressors, such as heterosexism and outness into interventions (Cochran, Peavy, \& Robohm, 2007). In conclusion, while previous research on sexually diverse individuals has shown that emotion dysregulation mediates the relation between instances of sexual minority status-based discrimination and alcohol use (Rogers et al., 2017), the present study is the first to extend that relation, albeit in a cross-sectional design, by showing how stress perceived from experiences of heterosexism, along with emotion dysregulation, may increase risk for harmful alcohol consumption as related to an LGB person's degree of outness. 


\section{References}

Aldao, A., Nolen-Hoeksema, S., \& Schweizer, S. (2010). Emotion-regulation strategies across psychopathology: A meta-analytic review. Clinical Psychology Review, 30, 217-237. https://doi.org/10.1016/j.cpr.2009.11.004

Allen, J. L., \& Mowbray, O. (2016). Sexual orientation, treatment utilization, and barriers for alcohol related problems: Findings from a nationally representative sample. Drug and Alcohol Dependence, 161, 323-330. https://doi.org/10.1016/j.drugalcdep.2016.02.025

Arnett, J. J. (2000). Emerging adulthood: A theory of development from the late teens through the twenties. American Psychologist, 55, 469-480. https://doi.org/10.1037//0003066X.55.5.469

Balsam, K. F., Beadnell, B., \& Molina, Y. (2013). The Daily Heterosexist Experiences Questionnaire - Measuring minority stress among LGBT adults. Measurement \& Evaluation in Counseling \& Development, 46(1), 3-25. https://doi.org/10.1177/0748175612449743.

Bjureberg, J., Ljótsson, B., Tull, M. T., Hedman, E., Sahlin, H., Lundh, G., .. Gratz, K. L. (2016). Development and validation of a brief version of the Difficulties in Emotion Regulation Scale: The DERS-16. Journal of Psychopathology and Behavioral Assessment, 38, 284-296. https://doi.org/10.1007/s10862-015-9514-x.

Bradford, J. B., Ryan, C., \& Rothblum, E. (1994). National Lesbian Health Care Survey: Implications for mental health. Journal of Consulting \& Clinical Psychology, 62, 228-242. https://doi.org/10.1037/0022-006X.62.2.228

Branscombe, N. R., \& Ellemers, N. (1998). Coping with group-based discrimination: Individualistic versus group-level strategies. Prejudice, 243-266. https://doi.org/10.1016/B978-012679130-3/50046-6 
Bryan, A. E. B., Kim, H., Fredriksen-Goldsen, K. I., Bryan, A. E. B., Kim, H., \& Fredriksengoldsen, K. I. (2017). Factors associated with high-risk alcohol consumption among LGB older adults: The roles of gender, social support, perceived stress, discrimination, and stigma. The Gerontologist, 57(S1), S95-104. https://doi.org/10.1093/geront/gnw100

Buhrmester, M., Kwang, T., \& Gosling, S. D. (2011). Amazon's Mechanical Turk: A new source of inexpensive, yet high-quality data? Prospectives on Psychological Science, 6, 3-5. https://doi.org/10.1177/1745691610393980

Burgard, S. A., Cochran, S. D., \& Mays, V. M. (2005). Alcohol and tobacco use patterns among heterosexually and homosexually experienced California women. Drug and Alcohol Dependence, 77, 61-70. https://doi.org/10.1016/j.drugalcdep.2004.07.007.Alcohol

Charak, R., Villarreal, L., Schmitz, R. M., Hirai, M., \& Ford, J. D. (2019). Patterns of childhood maltreatment and intimate partner violence, emotion dysregulation, and mental health symptoms among lesbian, gay, and bisexual emerging adults: A three-step latent class approach. Child Abuse and Neglect, 89, 99-110.

https://doi.org/10.1016/j.chiabu.2019.01.007

Charak, R., Byllesby, B. M., Fowler, J. C., Sharp, C., Elhai, J. D., \& Frueh, B. C. (2019). Assessment of the revised Difficulties in Emotion Regulation Scales among adolescents and adults with severe mental illness. Psychiatry Research, 279, 278-283. https://doi.org/10.1016/j.psychres.2019.04.010

Chow, P. K., \& Cheng, S. (2010). Shame, internalized heterosexism, lesbian identity, and coming out to others: A comparative study of lesbians in mainland China and Hong Kong, 57, 92-104. https://doi.org/10.1037/a0017930

Cochran, B. N., Peavy, K. M., \& Robohm, J. S. (2007). Do specialized services exist for LGBT 
individuals seeking treatment for substance misuse? A study of available treatment programs. Substance Use \& Misuse, 42, 161-176.

https://doi.org/10.1080/10826080601094207

Coker, T. R., Austin, S. B., \& Schuster, M. A. (2010). The health and health care of lesbian, gay, and bisexual adolescents. Annual Review of Public Health, 31, 457-477. https://doi.org/10.1146/annurev.publhealth.012809.103636

Cooper, M. L., Frone, M. R., Russell, M., \& Mudar, P. (1995). Drinking to regulate positive and negative emotions: A motivational model of alcohol use. Journal of Personality and Social Psychology, 69, 990-1005. https://doi.org/10.1037/0022-3514.69.5.990

Correro II, A. N., \& Nielson, K. A. (2019). A review of minority stress as a risk factor for cognitive decline in lesbian, gay, bisexual, and transgender ( LGBT ) elders. Journal of Gay \& Lesbian Mental Health. Advance online publication. https://doi.org/10.1080/19359705.2019.1644570

Coulter, R. W. S., Jun, H., Calzo, J. P., Truong, N. L., Mair, C., Markovic, N., .. Corliss, H. L. (2018). Sexual-orientation differences in alcohol use trajectories and disorders in emerging adulthood: results from a longitudinal cohort study in the United States. Addiction, 113, 1619-1632. https://doi.org/10.1111/add.14251

Craig, S. L., \& McInroy, L. (2014). You can form a part of yourself online: The influence of new media on identity development and coming out for LGBTQ youth. Journal of Gay \& Lesbian Mental Health, 18, 95-109. https://doi.org/10.1080/19359705.2013.777007

Dorn-Medeiros, C. M., \& Doyle, C. (2019). Alcohol as coping: Internalized homophobia and heterosexism's role in alcohol use among lesbians. Journal of LGBT Issues in Counseling, 12, 142-157. https://doi.org/10.1080/15538605.2018.1488230 
Dyar, C., Newcomb, M. E., \& Mustanski, B. (2019). Longitudinal associations between minority stressors and substance use among sexual and gender minority individuals. Drug and Alcohol Dependence, 201, 205-211. https://doi.org/10.1016/j.drugalcdep.2019.03.032

Esser, M. B., Hedden, S. L., Kanny, D., Brewer, R. D., Gfroerer, J. C., \& Naimi, T. S. (2014). Prevalence of Alcohol Dependence Among. Preventing Chronic Disease, 11(E206), 1-11.

Feinstein, B. A., Dyar, C., Li, D. H., Whitton, S. W., Newcomb, M. E., \& Mustanski, B. (2019). The longitudinal associations between outness and health outcomes among gay/lesbian versus bisexual emerging adults. Archives of Sexual Behavior, 48, 1111-1126. https://doi.org/10.1007/s10508-018-1221-8

Feinstein, B. A., Dyar, C., \& London, B. (2017). Are outness and community involvement risk or protective factors for alcohol and drug abuse among sexual minorityw? Archives of Sexual Behavior, 46, 1411-1423. https://doi.org/10.1007/s10508-016-0790-7

Feinstein, B. A., \& Newcomb, M. E. (2016). The role of substance use motives in the associations between minority stressors and substance use problems among young men who have sex with men. Psychology of Sexual Orientation and Gender Diversity, 3, 357-366. https://doi.org/10.1586/14737175.2015.1028369.

Fowler, J. C., Charak, R., Elhai, J. D., Allen, J. G., Frueh, B. C., \& Oldham, J. M. (2014). Construct validity and factor structure of the difficulties in Emotion Regulation Scale among adults with severe mental illness. Journal of Psychiatric Research, 58, 175-180. https://doi.org/10.1016/j.jpsychires.2014.07.029

Goldbach, J. T., Tanner-smith, E. E., Bagwell, M., \& Dunlap, S. (2014). Minority stress and substance use in sexual minority adolescents: A meta-analysis. Prevention Science, 15, 350-363. https://doi.org/10.1007/s11121-013-0393-7 
Gonzales, G., Przedworski, J., \& Henning-Smith, C. (2016). Comparison of health and health risk factors between lesbian, gay, and bisexual adults and heterosexual adults in the United States: Results from the national health interview survey. JAMA Internal Medicine, 176, 1344-1351. https://doi.org/10.1001/jamainternmed.2016.3432

Gratz, K. L., \& Roemer, L. (2004). Multidimensional assessment of emotion regulation and dysregulation: Development, factor structure, and initial validation of the Difficulties in Emotion Regulation Scale. Journal of Psychopathology and Behavioral Assessment, 26, $41-54$.

Gross, J. J. (2001). Emotion regulation in adulthood: Timing is everything. Current Directions in Psychological Science, 10, 214-219.

Gruskin, E. P., Hart, S., Gordon, N., \& Ackerson, L. (2001). Patterns of cigarette smoking and alcohol use among lesbians and bisexual women enrolled in a large health maintenance organization. American Journal of Public Health, 91, 976-979.

Hatzenbuehler, M. L. (2009). How does sexual minority stigma “Get Under the Skin”? A Psychological Mediation Framework, 135, 707-730. https://doi.org/10.1037/a0016441.How

Hatzenbuehler, M. L., Corbin, W. R., \& Fromme, K. (2011). Discrimination and alcohol-related problems among college students: A prospective examination of mediating effects. Drug and Alcohol Dependence, 115, 213-220. https://doi.org/10.1016/j.drugalcdep.2010.11.002

Hatzenbuehler, M. L., Nolen-hoeksema, S., \& Dovidio, J. (2009). How does stigma “"Get Under the Skin"'? The mediating role of emotion regulation. Psychological Science, 20, 12821289.

Hatzenbuehler, M. L., \& Pachankis, J. E. (2016). Stigma and minority stress as social determinants of health among lesbian, gay, bisexual, and transgender youth: Research 
evidence and clinical implications. Pediatric Clinics of North America, 63, 985-997. https://doi.org/10.1016/j.pcl.2016.07.003

Huebner, D. M., \& Davis, M. C. (2005). Gay and bisexual men who disclose their sexual orientations in the workplace have higher workday levels of salivary cortisol and negative affect. Annals of Behavioral Medicine, 30, 260-267.

https://doi.org/10.1207/s15324796abm3003_10

Hughes, T. (2011). Alcohol use and alcohol-related problems among sexual minority women. Alcoholism Treatment Quarterly, 29, 403-435. https://doi.org/10.1080/07347324.2011.608336

Juster, R. P., Smith, N. G., Ouellet, É., Sindi, S., \& Lupien, S. J. (2013). Sexual orientation and disclosure in relation to psychiatric symptoms, diurnal cortisol, and allostatic load. Psychosomatic Medicine, 75, 103-116. https://doi.org/10.1097/PSY.0b013e3182826881

Kanny, D., Naimi, T. S., Liu, Y., \& Lu, H. (2019). Annual total binge drinks consumed by U.S. adults, 2015. American Journal of Preventive Medicine, 54, 486-496. https://doi.org/10.1016/j.amepre.2017.12.021.

Kaysen, D., Kulesza, M., Balsam, K. F., Rhew, I. C., Blayney, J. A., Lehavot, K., \& Hughes, T. L. (2014). Coping as a mediator of internalized homophobia and psychological distress among young adult sexual minority women. Psychology of Sexual Orientation and Gender Diversity, 1, 225-233. https://doi.org/10.1037/sgd0000045

Kelly, J., Davis, C., \& Schlesinger, C. (2015). Substance use by same sex attracted young people: Prevalence, perceptions and homophobia. Drug and Alcohol Review, 34, 358-365. https://doi.org/10.1111/dar.12158

Khantzian, E. J. (1997). The self-medication hypothesis of substance use disorders: A 
reconsideration and recent applications. Harvard Review of Psychiatry, 4, 231-244. https://doi.org/10.3109/10673229709030550

King, M., Semlyen, J., Tai, S. S., Killaspy, H., Osborn, D., Popelyuk, D., \& Nazareth, I. (2008). A systematic review of mental disorder, suicide, and deliberate self harm in lesbian, gay and bisexual people. BMC Psychiatry, 8, 70. https://doi.org/10.1186/1471-244X-8-70

Kosciw, J. G., Greytak, E. A., Bartkiewicz, M. J., Boesen, M. J., \& Palmer, N. A. (2012). The 2011 National School Climate Survey: The Experiences of Lesbian, Gay, Bisexual and Transgender Youth in Our Nation's Schools.

Kosciw, J. G., Palmer, N. A., \& Kull, R. M. (2014). Reflecting resiliency: Openness about sexual orientation and/or gender identity and its relationship to well-being and educational outcomes for LGBT students. American Journal of Community Psychology, 55, 167-178. https://doi.org/10.1007/s10464-014-9642-6

Lee, J. H., Gamarel, K. E., Bryant, K. J., Zaller, N. D., \& Operario, D. (2016). Discrimination, mental health, and substance use disorders among sexual minority populations. $L G B T$ Health, 3, 258-265. https://doi.org/10.1089/lgbt.2015.0135

Legate, N., Ryan, R. M., \& Weinstein, N. (2012). Is coming out always a "good thing”? exploring the relations of autonomy support, outness, and wellness for lesbian, gay, and bisexual individuals. Social Psychological and Personality Science, 3, 145-152. https://doi.org/10.1177/1948550611411929

Lewis, R. J., Mason, T. B., Winstead, B. A., Gaskins, M., \& Irons, L. B. (2016). Pathways to hazardous drinking among racially and socioeconomically diverse lesbian women: Sexual minority stress, rumination, social isolation, and drinking to cope. Psychology of Women Quarterly, 40, 564-581. https://doi.org/10.1177/0361684316662603 
Livingston, Ni. A., Christianson, N., \& Cochran, B. N. (2016). Minority stress, psychological distress, and alcohol misuse among sexual minority young adults: A resiliency-based conditional process analysis. Addictive Behaviors, 63, 125-131. https://doi.org/10.1016/j.addbeh.2016.07.011

Macapagal, K., Bhatia, R., \& Greene, G. J. (2016). Differences in healthcare access, use, and experiences within a community sample of racially diverse lesbian, gay, bisexual, transgender, and questioning emerging adults. LGBT Health, 3, 434-442. https://doi.org/10.1089/lgbt.2015.0124

Mackinnon, D. P., Lockwood, C. M., \& Williams, J. (2004). Confidence limits for the indirect effect: Distribution of the product and resampling method. Multivariate Behavioral Research, 39, 99-128. https://doi.org/10.1207/s15327906mbr3901

Mays, V. M., \& Cochran, S. D. (2001). Mental health correlates of perceived discrimination among lesbian, gay, and bisexual adults in the United States. American Journal of Public Health, 91, 1869-1876.

McCabe, S. E., Bostwick, W. B., Hughes, T. L., West, B. T., \& Boyd, C. J. (2010). The relationship between discrimination and substance use disorders among lesbian, gay, and bisexual adults in the United States. American Journal of Public Health, 100, 1946-1952. https://doi.org/10.2105/AJPH.2009.163147

McLaughlin, K. A., Hatzenbuehler, M. L., \& Hilt, L. M. (2009). Emotion dysregulation as a mechanism linking peer victimization to internalizing symptoms in adolescents. Journal of Consulting \& Clinical Psychology, 77, 894-904. https://doi.org/10.1037/a0015760.Emotion

McLaughlin, K. A., Hatzenbuehler, M. L., Mennin, D. S., \& Nolen-Hoeksema, S. (2011). Emotion dysregulation and adolescent psychopathology: A prospective study. Behaviour 
Research and Therapy, 49, 544-554. https://doi.org/10.1016/j.brat.2011.06.003

Meidlinger, P. C., \& Hope, D. A. (2014). Differentiating disclosure and concealment in measurement of outness for sexual minorities: The Nebraska outness Scale. Psychology of Sexual Orientation and Gender Diversity, 1, 489-497. https://doi.org/10.1037/sgd0000080

Meyer, I. H. (2003). Prejudice, social stress, and mental health in lesbian, gay, and bisexual populations: Conceptual issues and research evidence. Psychological Bulletin, 129, 674697. https://doi.org/10.1016/j.dcn.2011.01.002.

Meyer, I. H., Schwartz, S., \& Frost, D. M. (2008). Social patterning of stress and coping: Does disadvantaged social status confer more stress and fewer coping resources? Social Science Medicine, 67, 368-379. https://doi.org/10.1016/j.socscimed.2008.03.012.

Miguel, F. K., Giromini, L., Colombarolli, S., Zuanazzi, A. C., \& Zennaro, A. (2017). A brazilian investigation of the 36- and 16-Item Difficulties in Emotion Regulation Scales. Journal of Clinical Psychology, 73, 1146-1159. https://doi.org/10.1002/jclp.22404

Morris, J. F., Waldo, C. R., \& Rothblum, E. D. (2001). A model of predictors and outcomes of outness among lesbian and bisexual women. American Journal of Orthopsychiatry, 71, 6171. https://doi.org/10.1037/0002-9432.71.1.61

Ngamake, S. T., Walch, S. E., \& Raveepatarakul, J. (2016). Discrimination and sexual minority mental health: Mediation and moderation effects of coping. Psychology of Sexual Orientation and Gender Diversity, 3, 213-226. https://doi.org/10.1037/sgd0000163

Nolen-Hoeksema, S., \& Harrell, Z. A. (2002). Rumination, depression, and alcohol use: Tests of gender differences. Journal of Cognitive Psychotherapy, 16, 391-403. https://doi.org/10.1891/jcop.16.4.391.52526

Pachankis, J. E., Cochran, S. D., \& Mays, V. M. (2015). The mental health of sexual minority 
adults in and out of the closet: A population-based study. Journal of Consulting \& Clinical Psychology, 83, 890-901. https://doi.org/10.1186/s40945-017-0033-9.

Pachankis, J. E., Rendina, J. H., Restar, A., Ventuneac, A., Grov, C., \& Parsons, J. T. (2015). A minority stress - emotion regulation model of sexual compulsivity among highly sexually active gay and bisexual men. Health Psychology, 34, 829-840. https://doi.org/10.1037/hea0000180.A

Pakula, B., Shoveller, J., Ratner, P. A., \& Carpiano, R. (2016). Prevalence and co-occurrence of heavy drinking and anxiety and mood disorders among gay, lesbian, bisexual, and heterosexual Canadians. American Journal of Public Health, 106, 1042-1048. https://doi.org/10.2105/AJPH.2016.303083

Preacher, K. J., \& Hayes, A. F. (2008). Asymptotic and resampling strategies for assessing and comparing indirect effects in multiple mediator models. Behavior Research Methods, 40, 879-891. https://doi.org/10.3758/BRM.40.3.879

Riggle, E. D. B., Rostosky, S. S., Black, W. W., \& Rosenkrantz, D. E. (2017). Outness, concealment, and authenticity: Associations with LGB individuals' psychological distress and well-being. Psychology of Sexual Orientation and Gender Diversity, 4(1), 54-62. https://doi.org/10.1037/sgd0000202

Rogers, A. H., Seager, I., Haines, N., Hahn, H., Aldao, A., Costa, A. B., .. Rogers, A. H. (2017). The Indirect Effect of Emotion Regulation on Minority Stress and Problematic Substance Use in Lesbian, Gay, and Bisexual Individuals. Frontiers in Psychology, 8, 1881. https://doi.org/10.3389/fpsyg.2017.01881

Rosario, M., Schrimshaw, E. W., \& Hunter, J. (2009). Disclosure of sexual orientation and subsequent substance use and abuse among lesbian, gay, and bisexual youths: Critical role 
of disclosure reactions. Psychology of Addictive Behaviors, 23(1), 175-184. https://doi.org/10.1037/a0014284.Disclosure

Russell, S. T., Toomey, R. B., Ryan, C., \& Diaz, R. M. (2014). Being Out at School: The Implications for School Victimization and Young Adult Adjustment. American Journal of Orthopsychiatry, 84(6), 635-643.

Saunders, J. B., Aasland, O. G., Babor, T. F., Fuente, J. R. D. E. L. A., Grant, M., \& Saunders, J. B. (1993). Development of the Alcohol Use Disorders Identification Test (AUDIT): WHO Collaborative Project on Early Detection of Persons with Harmful Alcohol Consumption II, 88(6), 791-804.

Schmitz, R. M., Robinson, B. A., Tabler, J., Welch, B., \& Rafaqut, S. (2019). LGBTQ+ Latino/a Young People's Interpretations of Stigma and Mental Health: An Intersectional Minority Stress Perspective. Society and Mental Health. https://doi.org/10.1177/2156869319847248

Schmitz, R. M., \& Tyler, K. A. (2018a). Contextual constraints and choices: Strategic identity management among LGBTQ youth. Journal of LGBT Youth, O(0), 1-15. https://doi.org/10.1080/19361653.2018.1466754

Schmitz, R. M., \& Tyler, K. A. (2018b). LGBTQ + Young Adults on the Street and on Campus : Identity as a Product of Social Context LGBTQ + Young Adults on the Street and on Campus : Journal of Homosexuality, 65(2), 197-223. https://doi.org/10.1080/00918369.2017.1314162

Schmitz, R. M., \& Tyler, K. A. (2019). 'Life has actually become more clear': An examination of resilience among LGBTQ young adults. Sexualities, 22(4), 710-733. https://doi.org/10.1177/1363460718770451

Shadur, J. M., \& Lejuez, C. W. (2015). Adolescent Substance Use and Comorbid 
Psychopathology: Emotion Regulation Deficits as a Transdiagnostic Risk Factor. Current Addiction Reports, 2(4), 354-363. https://doi.org/10.1007/s40429-015-0070-y

Shapiro, D. N., Chandler, J., \& Mueller, P. A. (2013). Using Mechanical Turk to Study Clinical Populations. Clinical Psychological Science, 1(2), 213-220. https://doi.org/10.1177/2167702612469015

Simoni, J. M., \& Walters, K. L. (2001). Heterosexual Identity and Heterosexism. Journal of Homosexuality, 41(1), 157-172. https://doi.org/10.1300/J082v41n01

Swim, J. K., Johnston, K., \& Pearson, N. B. (2009). Daily experiences with heterosexism: Relations between heterosexist hassles and psychological well-being. Journal of Social and Clinical Psychology, 28(5), 597-629.

Szymanski, D. M. (2009). Examining Potential Moderators of the Link Between Heterosexist Events and Gay and Bisexual Men’s Psychological Distress. Journal of Counseling Psychology, 56(1), 142-151. https://doi.org/10.1037/0022-0167.56.1.142

Szymanski, D. M., \& Mikorski, R. (2016). External and Internalized Heterosexism, Meaning in Life, and Psychological Distress. Psychology of Sexual Orientation and Gender Diversity, $3(3), 265-274$.

Thomas, K. A., \& Clifford, S. (2017). Computers in Human Behavior Validity and Mechanical Turk: An assessment of exclusion methods and interactive experiments. Computers in Human Behavior, 77, 184-197. https://doi.org/10.1016/j.chb.2017.08.038

Tice, D. M., Bratslavsky, E., \& Baumeister, R. F. (2001). Emotional distress regulation takes precedence over impulse control: If you feel bad, do it! Journal of Personality and Social Psychology, 80(1), 53-67. https://doi.org/10.1037/0022-3514.80.1.53

Veilleux, J. C., Skinner, K. D., Reese, E. D., \& Shaver, J. A. (2014). Negative affect intensity 
influences drinking to cope through facets of emotion dysregulation. Personality and Individual Differences, 59, 96-101. https://doi.org/10.1016/j.paid.2013.11.012

Velez, B. L., \& Moradi, B. (2016). A Moderated Mediation Test of Minority Stress. The Counseling Psychologist, 44(8), 1132-1157. https://doi.org/10.1177/0011000016665467

Walters, K., Christakis, D. A., \& Wright, D. R. (2018). Are Mechanical Turk worker samples representative of health status and health behaviors in the U.S.? PLoS ONE, 13(6), e0198835. https://doi.org/10.7910/DVN/I7U3GT

Whitman, C. N., \& Nadal, K. L. (2015). Sexual Minority Identities: Outness and Well-Being Among Lesbian, Gay, and Bisexual Adults. Journal of Gay \& Lesbian Mental Health, 19(4), 370-396. https://doi.org/10.1080/19359705.2015.1038974

Williams, T., Connolly, J., Pepler, D., \& Craig, W. (2005). Peer victimization, social support, and psychosocial adjustment of sexual minority adolescents. Journal of Youth and Adolescence, 34(5), 471-482. https://doi.org/10.1007/s 10964-005-7264-X

Wilsnack, S. C., Hughes, T. L., Johnson, T. P., Bostwick, W. B., Szalacha, L. A., Benson, P., ... Kinnison, K. E. (2008). Drinking and Drinking-Related Problems Among Heterosexual and Sexual Minority Women. Journal of Studies on Alcohol and Drugs, 69(1), 129-139. 
Table 1

Correlation between outness, heterosexist experiences, emotion dysregulation and alcohol use among lesbian, gay, and bisexual individuals

\begin{tabular}{lllll}
\hline Variables & Outness & HE & DERS & Alcohol use \\
\hline Outness & 1 & $-.163^{* *}$ & $-.349^{* *}$ & -.024 \\
HE & & 1 & $.443^{* *}$ & $.320^{* *}$ \\
DERS & & & 1 & $.244^{* *}$ \\
Alcohol use & & & & 1 \\
\hline
\end{tabular}

Note. $\mathrm{HE}=$ Heterosexist experiences. DERS $=$ Difficulties in emotion regulation.

$* * p<.01$. 
Table 2

Significant indirect effects in the association between outness, heterosexist experiences, emotion dysregulation, and alcohol use among lesbian, gay, and bisexual individuals

\begin{tabular}{|c|c|c|c|}
\hline \multicolumn{2}{|c|}{ Pathways } & \multirow{2}{*}{$\begin{array}{l}\text { Indirect effect }(B) \\
-.008\end{array}$} & \multirow{2}{*}{$\begin{array}{l}\text { Indirect effect }(95 \% \mathrm{CI}) \\
-.016 \text { to }-.002\end{array}$} \\
\hline 1 & Outness $\rightarrow$ HE $\rightarrow$ Alcohol use (M1) & & \\
\hline 2 & Outness $\rightarrow$ DERS $\rightarrow$ Alcohol use (M2) & -.008 & -.015 to -.003 \\
\hline \multirow[t]{5}{*}{3} & Outness $\rightarrow \mathrm{HE} \rightarrow$ DERS $\rightarrow$ Alcohol use (M3) & -.002 & -.004 to -.0004 \\
\hline & & Estimates & \\
\hline & M1 vs. M2 & $.001 \mathrm{~ns}$ & -.011 to .010 \\
\hline & M1 vs. M3 & $.006^{*}$ & $\begin{array}{l}-.014 \text { to }-.001 \\
\mathrm{M} 3>\mathrm{M} 1\end{array}$ \\
\hline & M2 vs. M3 & $.006 *$ & $\begin{array}{l}-.012 \text { to }-.002 \\
\mathrm{M} 3>\mathrm{M} 2\end{array}$ \\
\hline
\end{tabular}

Note . HE $=$ Heterosexist experiences. DERS $=$ Difficulties in emotion regulation. $* p<0.05$. 


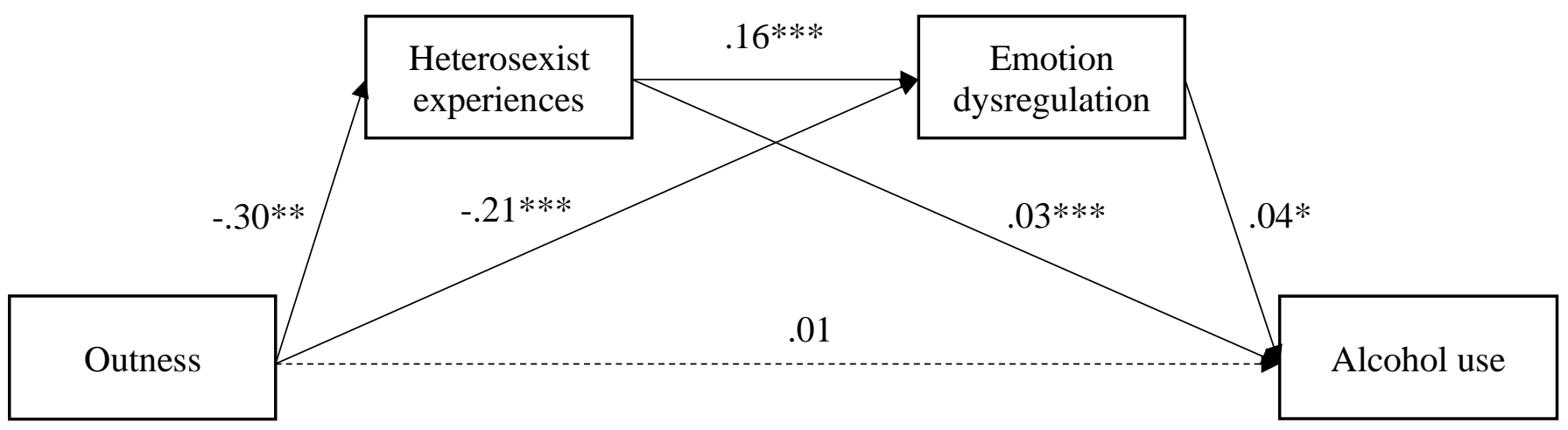

Figure 1.

Unstandardized coefficients $(B)$ of direct and mediated pathways between outness and alcohol use, with heterosexist experiences and emotion dysregulation as mediators.

All significant pathways $(p<.05)$ are represented by a bold line, and non-significant pathways by a dashed line. All error terms of the mental health outcomes are intercorrelated.

$* p<.05$

$* * p<.01$

$* * * p<.001$ 